\title{
Sobre los paradigmas de la violencia
}

\section{About paradigms of violence}

\author{
Marcos Pablo Moloeznik• \\ Radamanto Portilla-Tinajero*•
}

\section{Resumen}

La violencia constituye un fenómeno complejo a la vez que una constante histórica. Esto explica que diversos pensadores y tratadistas hayan intentado definirla, interpretarla y clasificarla. La violencia da al traste con el más sagrado de los derechos humanos, el derecho a la vida. En particular, el genocidio y otros crímenes de lesa humanidad, los crímenes de guerra, los actos esporádicos y aislados de violencia - otras situaciones de violencia-, el terrorismo y la violencia criminal organizada, son las modalidades de la violencia colectiva responsables de la mayoría de las muertes violentas que cuestionan severamente el pacto social. De ahí la imperiosa necesidad de llevar a cabo una revisión de los paradigmas de la violencia y de los tipos de violencia más perniciosos y que causan mayor daño al tejido social.

Palabras clave: violencia, genocidio, conflicto armado, otras situaciones de violencia (osv), terrorismo, violencia criminal organizada.

\begin{abstract}
Violence constitutes a complex phenomenon as well as an historical constant. This explains that various thinkers and writers have tried to define, interpret and classify it. Violence destroys the most sacred of human rights, the right to life. In particular, genocide and other crimes against humanity, war crimes, sporadic and isolated acts of violence - other situations of violence-, terrorism and organized criminal violence, are the modalities of collective violence responsible for majority of the violent deaths that severely question the social pact. Hence the urgent need to carry out a review of the paradigms of violence and the most pernicious types of violence that cause the greatest damage to the social dimension.
\end{abstract}

Keywords: violence, genocide, armed conflicts, other situations of violence (osv), terrorism, organized criminal violence.

- Doctor en Derecho por la Universidad de Alcalá, España. Profesor-investigador adscrito al Centro Universitario de Ciencias Sociales y Humanidades, de la Universidad de Guadalajara, México. Profesor huésped del Instituto Internacional de Derecho Humanitario, Sanremo, Italia. Primer académico no estadounidense en obtener el Premio William J. Perry a la Excelencia en Educación en Seguridad y Defensa,William J. Perry Center for Hemispheric Defense Studies, National Defense University, Washington, dc. Orcid: 0000-0002-4078-945I / mmoloeznik@csh.udg.mx

- Doctor en Ciencias Sociales por la Universidad de Guadalajara. Profesor de asignatura en el Instituto Tecnológico de Estudios Superiores de Occidente, Universidad Jesuita de Guadalajara; consultor de la Oficina de las Naciones Unidas contra la Droga y el Delito; director de Evaluación de Políticas Públicas en el Gobierno del Estado de Jalisco. Líneas de investigación: violencia criminal, evaluación de políticas públicas, políticas basadas en evidencia. Orcid: 0000-000I-55971072 /_radamanto@iteso.mx

Fecha de recepción: 8 de septiembre de 2020. Fecha de aceptación: 4 de junio de 202I. 
Introducción

La violencia, en sus diversas manifestaciones, es el signo de los tiempos. En especial, la humanidad se ve compelida - de manera permanente- por la violencia colectiva que se presenta bajo las modalidades de genocidio, crímenes de lesa humanidad, crímenes de guerra y violencia criminal organizada, que afectan a grandes colectivos sociales. ${ }^{1}$ Esto es así, dado que "Los conflictos son connaturales a la especie humana. Nuestra ley originaria es la ley de la selva, donde las relaciones están fundadas en la fuerza, y, en todo caso, en la pertenencia a la manada [...]" (Marina, 2004: 155).

En otros términos, la propia realidad signada por la violencia impone un análisis de los diferentes paradigmas que intentan explicar la complejidad de dicho fenómeno. Para ello, el trabajo que se pone a consideración del lector se divide en las siguientes secciones: (a) un recorrido por las diferentes definiciones de la violencia; (b) una revisión de la tipología de la misma; (c) la aprehensión del "crimen de los crímenes": el genocidio; (d) el conflicto armado como constante histórica; (e) lo que el Comité Internacional de la Cruz Roja (1998) denomina "otras situaciones de violencia", cada vez más comunes en América Latina; (f) el terrorismo responsable de ataques indiscriminados contra la población civil, y (g) el flagelo de la violencia criminal organizada, que reconoce a la región como la más violenta del mundo.

En síntesis, se trata de un esfuerzo científico de sistematización de información para dar cuenta de los diferentes paradigmas y modalidades de la violencia.

I. Para dar cuenta de esta realidad insoslayable, se parte de los contenidos desarrollados a lo largo de los sucesivos Cursos Avanzados en Derecho Internacional Humanitario en idioma español - de 2013 a 2019, inclusive- en el Instituto Internacional de Derecho Humanitario de Sanremo, Italia, del autor en calidad de profesor visitante, así como hallazgos de la tesis doctoral del coautor. 


\section{Violencia}

En primer lugar, cabe destacar que no existe una definición de la violencia aceptada universalmente, así como tampoco una teoría que sea capaz de explicar todas las formas de violencia (Bernstein, 2015) no obstante los intentos de reconocidos filósofos, sociólogos y antropólogos (véase Arendt, 2006; Benjamin, 1995; Collins, 2008; Gros, 2006; Schinkel, 2010; Sofsky, 2004; Sorel, 2004; Tilly, 2008; Wieviorka, 2009; Zizek, 2009, entre otros). Lo paradójico, de acuerdo con Bernstein (2015: 31), "es que a pesar de (o quizás debido a) que haya tanta discusión sobre la violencia, existe una enorme confusión respecto a qué entendemos por violencia”.

Así, no se debe soslayar que cualquier definición o tipología de la violencia incluirá algunas de sus expresiones o modalidades y, necesariamente, excluirá otras. Este problema radica en la imposibilidad de incluir, en un mismo concepto, todos los actos considerados como violencia según las distintas culturas, momentos históricos e intereses analíticos.

No obstante este problema, básicamente es posible distinguir dos concepciones generales de la violencia: una restringida y otra ampliada (véase Arteaga Botello, 2007; Blair, 2009; Crettiez, 2009). La concepción restringida de la violencia enfatiza en su característica objetiva y medible el daño físico. Desde esta perspectiva, se define a la violencia como el uso de la fuerza física, directa y excesiva, con el objetivo de causar daño a otro (véase García-Sílberman y Ramos Lira, 2000: 13-79). En esta concepción la muerte ajena a causas naturales se constituye como el indicador global y predilecto de la violencia, y se pone especial énfasis en las consecuencias y la gravedad del daño sobre las víctimas.

Por su parte, la concepción ampliada de la violencia reconoce un conjunto más amplio de actos que implican ejercicios 
intencionales de poder contra individuos o colectivos, que no se expresan solamente en daños físicos, pero que reproducen relaciones sistemáticas de desigualdad, exclusión, subordinación u otras formas sutiles de victimización y coerción (Crettiez, 2009; Gros, 2006; Kaldor, 2001). Desde esta perspectiva es posible hablar de violencia política (Rouquié, 1989; Schmitt, 2014; Tilly, 2008), violencia estructural (Fanon, 1973; Galtung, 1998), violencia totalitaria (Arendt, 1967, 2006), violencia simbólica (Bourdieu y Passeron, 2001; Zizek, 1999, 2009), violencia cultural (Galtung, 2009; Zizek, 2009) e, incluso, violencia legal (Benjamin, 1995).

\section{Tipología de la violencia}

En segundo término, existen diferentes formas de clasificar la violencia y, dependiendo de los propósitos analíticos, es posible proponer diferentes esquemas explicativos (Bernstein, 2015: 260). En este marco se presenta una propuesta de clasificación de la violencia que enfatiza en la concepción restringida, a partir de cuatro criterios: a) los actores de la violencia; b) los motivos de la violencia; c) el grado de organización de la violencia, y d) los escenarios de la violencia.

Esta clasificación, si bien limitada, permite identificar las características sustantivas de la violencia criminal organizada, responsable del mayor número de homicidios dolosos en Latinoamérica.

\section{Desde los actores de la violencia}

La Organización Panamericana de la Salud adopta la concepción restrictiva en su informe sobre violencia y salud, y la define como

[...] el uso intencional de la fuerza o el poder físico, de hecho o como amenaza, contra uno mismo, otra persona o un grupo o comunidad, que cause $o$ tenga muchas probabilidades de causar lesiones, muerte, daños 
psicológicos, trastornos del desarrollo o privaciones (Organización

Panamericana de la Salud (ops), 2003:5).

A partir de esta concepción genera una tipología de la violencia de acuerdo con el actor o actores que cometen o son víctimas del acto violento: violencia autoinfligida, violencia interpersonal y violencia colectiva.

La violencia autoinfligida corresponde a los actos que una persona se inflige a sí misma; por ejemplo, el suicidio y las autolesiones. La violencia interpersonal es aquella en la que un individuo o un grupo pequeño de individuos cometen actos violentos contra otro individuo. Este tipo de violencia contiene un gran número de formas de expresión, que van desde el maltrato infantil hasta el homicidio. La violencia interpersonal, a su vez, se subdivide según el grado de cercanía social de la víctima y el victimario. Cuando ambos tienen un grado de parentesco se le conoce como violencia familiar o de pareja; cuando la violencia se produce entre personas que no guardan parentesco y que pueden conocerse o no, y sucede regularmente fuera del hogar, se le llama violencia comunitaria. Por último, se conoce como violencia colectiva aquellos actos que afectan a individuos, grupos sociales o colectivos. Generalmente la violencia colectiva la infligen grandes grupos organizados, como el Estado, contingentes políticos, fuerzas militares irregulares y organizaciones terroristas (ops, 2003). La guerra, los actos de odio, el terrorismo, los conflictos armados, las masacres, son formas de la violencia del tipo colectivo (oPs, 2003).

La importancia de esta tipología de la violencia, propuesta por la Organización Mundial de la Salud a través de su oficina regional para las Américas (la ops), radica en dos aspectos: primero, genera un conjunto de indicadores que facilitan la medición de la magnitud de la violencia y su comparación a nivel local, regional e internacional; y segundo, el enfoque de la violencia desde la perspectiva 
de la salud posibilita la interdisciplinariedad y la acción colectiva en la solución del problema (ops, 2003: 4).

Para la ops, los datos sobre la mortalidad "pueden proporcionar un indicio del grado de violencia mortal en una comunidad o un país determinado" (ops, 2003: 8); por ello, las tasas por cada 100 mil habitantes de homicidios, suicidios, bajas relacionadas con la guerra, etc., representan los principales indicadores para la medición de la violencia (World Health Organization, 2014). Por otro lado, el enfoque de la violencia desde la perspectiva de la salud se basa en el método científico, para lo que es necesaria la recopilación sistemática de información, la identificación de las causas o factores que producen la violencia, el análisis de la relación entre los factores y la definición de acciones efectivas que prevengan la violencia. En particular, el enfoque de la salud concede suma importancia a la prevención (oPs, 2003: 5).

Es justo señalar también las limitaciones de la tipología de la violencia propuesta por la ops. En primer lugar, quizá la más importante, es la limitada concepción de la violencia, al reducirla a la expresión física de la misma. La violencia no siempre se expresa físicamente, con lesiones, muerte, daños o trastornos. Por otro lado, la violencia se expresa de formas complejas que no pueden ser abarcadas solamente por la identificación de los agentes generadores. Sin embargo, la identificación de los actores de la violencia es un buen principio para generar una tipología de la violencia.

\section{Las razones de la violencia}

Clasificar la violencia tomando de referencia las razones o los motivos de los actores de la violencia es un arduo trabajo: habría tantas razones como individuos que la ejerzan. Pero, de enfocarse solamente en aquellos actores colectivos, es posible identificar ciertas generalidades. De ahí que se propone, en un primer momento, tres grandes razones o motivaciones de la violencia: económicas, políticas 
y socioculturales. Evidentemente los actores de la violencia pueden tener más de un tipo de razones (motivos múltiples), combinarlas en un momento determinado (motivos mixtos), o tener motivos ocultos o poco evidentes, por lo que se incluyen en esta clasificación.

Cuando los actos de la violencia son motivados por la obtención de recursos económicos o materiales, se clasifica como violencia económica. Desde esta perspectiva, el comportamiento de los actores de la violencia se concibe como económicamente racional, es decir, actúan de determinada manera con el objeto de obtener un beneficio económico o material. Éstos pueden ser objetivos de cualquier grupo o colectivo, como el Estado, las guerrillas, los terroristas, aunque suele atribuírsele principalmente a los delincuentes y actores del crimen organizado (Bagley y Rosen, 2015; Beittel, 2015; Siegel y Bunt, 2012). Ésta suele ser una de las explicaciones más convencionales del ejercicio de la violencia por este tipo de actores no estatales, pero no se limita a ellos. Algunos estudios han mostrado cómo, por ejemplo, las guerrillas colombianas (Restrepo y Aponte, 2009) o los extremistas islámicos (Forst, 2008) han recurrido a la violencia para hacerse de recursos que den sostenibilidad a sus movimientos.

La violencia política comprende todos aquellos actos de violencia que tienen como fin el mantenimiento del orden público, el respeto a las leyes o la adquisición de poder. Este tipo de violencia ha sido principalmente estudiado en actores del Estado, grupos guerrilleros, movimientos sociales y terroristas (véase Imbusch, Misse y Carrión, 2011; Tilly, 2008; Winkler, 2006). No obstante, el ejercicio de la violencia de parte de algunos grupos del crimen organizado ha mostrado fines políticos, aunque no busquen tomar el poder político o cambiar el sistema político, si tienen incidencia en las decisiones de las autoridades, en las políticas públicas, 
en la generación de bienes públicos o en el control de facto de funciones gubernamentales (Lessing, 2012; Schedler, 2015). ${ }^{2}$

Por último, se habla de violencia social o cultural cuando aspectos de la cultura o del orden simbólico — por ejemplo la religión y la ideología, el lenguaje y el arte, la ciencia formal, la lógica y el razonamiento- son empleados para justificar actos violentos (véase Galtung, 1998, 2009). Este tipo de violencia se expresa en la desigualdad económica, en el racismo, en las diferencias de género, en el acceso a los servicios públicos, en las posibilidades de desarrollo humano y consecución del bienestar.

Como se menciona en párrafos anteriores, los motivos de los actores de la violencia no son excluyentes, éstos pueden apelar a distintas razones al momento del ejercicio de la violencia. Tal como lo considera Schedler (2015: 54): "la atribución de motivos es estructuralmente difícil". ¿Cómo sabemos cuáles son los "motivos verdaderos" de los actores de la violencia? La mayoría de las personas, incluidos políticos y académicos, suelen pensar que los motivos de los grupos criminales son exclusivamente económicos. Pero, ¿cómo estar tan seguro de ello? Por ejemplo, algunos grupos criminales como la Familia Michoacana o los Caballeros Templarios en México han dado muestras claras de intereses políticos, incluso llegando a suplantar algunas funciones del gobierno y de las autoridades (Maldonado Aranda, 2012). En el caso de los Caballeros Templarios los motivos religiosos han formado parte de su agenda criminal (Beittel, 2015; Reguillo, 2014). Esto invita a pensar en la dificultad de atribuir determinantemente motivaciones a los

2. De acuerdo con Tilly $(2006,2008)$, la violencia colectiva es siempre política: las interacciones se dan entre colectivos que comparten un conjunto de valores, normas, intereses en común; mediante esta interacción un colectivo intenta imponer sus valores, normas o intereses al otro colectivo; regularmente el Estado, las autoridades legales o los actores gubernamentales son uno de los colectivos que participan en la interacción. Por estas razones resulta pertinente desarrollar con mayor profundidad las categorías de violencia colectiva y violencia política. 
actores violentos, ya que cabe la posibilidad de existir motivos ocultos que sean difíciles de inferir de manera directa.

Por otro lado, es más común que en la violencia criminal organizada se identifiquen motivos mixtos o múltiples. En otras palabras, seguramente la principal motivación de los actores de la violencia criminal organizada sea la obtención de recursos o la maximización de utilidades pero, como ha argumentado Kalyvas $(2006,2015)$ en sus estudios sobre diferentes formas de guerra civil, la selección de las víctimas, las formas de la violencia y el tiempo en el que se ejerce regularmente está motivada por asuntos políticos, culturales o personales — por ejemplo, por motivos personales como la envidia, el resentimiento, la competencia amorosa o los rencores familiares suelen elegirse muchas de las personas asesinadas por el crimen organizado-. En este sentido, una explicación puramente desde la racionalidad económica de los actores limita la comprensión de la violencia criminal organizada.

\section{Grado de organización de la violencia}

Distinguir la violencia según su grado de organización es pertinente porque habla de toda una maquinaria puesta en función de la administración de la muerte (véase Mbembe, 2011; Valencia, 2010). Esta distinción marca una diferencia respecto al "delito común" y permite enfocarse en la violencia que se manifiesta de manera organizada, la cual es característica de la violencia efectuada por colectivos como el crimen organizado o el Estado. En este marco, solamente es posible hablar de violencia organizada cuando se trata de actos realizados por colectivos fuertemente estructurados, con gran autonomía operativa y alta diferenciación interna (jerarquía) y externa (membresía) (véase Malesevic, 2010; Schedler, 2015; Tilly, 2008).

En cambio, la violencia desorganizada, oportunista o casuística, no menos maligna que la violencia organizada, es 
producto de individuos o de colectivos donde el acto violento parece haber sido más una consecuencia de sus acciones que un medio para un fin. En este tipo de violencia los actores violentos no están coordinados, carecen de mecanismos o procesos sistemáticos para el ejercicio de la violencia, están más determinados por la situación o el contexto (oportunidad) y las motivaciones personales (véase Collins, 2008, 2009). Cuando existe cierto grado de sistematicidad, organización y prevalencia de los actos violentos, éstos son realizados generalmente a título personal, como podrían ser los feminicidios o los asesinatos en serie.

\section{Los escenarios de la violencia}

Otra manera de clasificar la violencia es atendiendo a los escenarios espaciales y temporales en los que se manifiesta. Uno de estos tipos de violencia es la situacional, la que se desarrolla en el espacio de la vida cotidiana. Ésta ha sido estudiada, entre otros, por Zimbardo (2008) y Collins $(2008,2009)$. La premisa fundamental es que la emergencia de la violencia está condicionada por situaciones sociales concretas, es decir, por la conjunción de diversos elementos institucionales, simbólicos y personales que se activan en la interacción social entre los individuos o grupos en disputa, en un momento y espacio determinado. Debido a la complejidad que esto implica, la perspectiva situacional de la violencia considera que los actos de violencia manifiestos son una excepción en la infinidad de interacciones sociales conflictivas que se resuelven sin violencia. Esta perspectiva permite explicar la gran mayoría de actos violentos individuales, como los homicidios y las agresiones, así como algunos actos violentos colectivos, como las peleas callejeras de las pandillas, las agresiones en eventos deportivos o públicos, o los enfrentamientos entre fuerzas armadas y la delincuencia organizada. 
Otros escenarios a considerar cuando se habla de violencia organizada son las situaciones complejas de emergencia y los conflictos armados. Una situación compleja de emergencia es, de acuerdo con las Naciones Unidas, "una crisis humanitaria en un país, región o sociedad, donde hay un deterioro total o considerable de la autoridad como resultado de conflictos internos o externos, que requieren una respuesta internacional [...]" (ops, 2003: 235). El término se utiliza para describir situaciones de violencia sistemática y prevalente que tienen implicaciones políticas internas o externas, en las cuales parece necesaria una intervención de organismos internacionales. ${ }^{3}$

En lo que respecta a los conflictos armados, se hace una distinción entre dos tipos de conflictos armados: (a) los conflictos armados internacionales (CAI), que tienen lugar entre dos Estados o más, y (b) los conflictos armados no internacionales, (CANI) que se libran entre Estados y grupos armados no gubernamentales, o entre estos grupos únicamente (Melzer, 2019: 57). Dicho en otras palabras, se reconoce la existencia de un CANI "cuando ocurre una situación de violencia armada prolongada entre las autoridades gubernamentales y un grupo armado organizado o más, o entre esos grupos" (Melzer, 2019: 55). Lo que es retomado por Schedler (2015: 49), para quien se trata de "una confrontación entre grupos armados dentro de un Estado, o entre un grupo armado y el mismo Estado, que causa un mínimo de mil muertos al año".

Este tipo de escenarios permite comprender la violencia organizada bajo lógicas que trascienden las motivaciones económicamente racionales que se le atribuyen a la violencia criminal organizada que ejercen los grupos del crimen

3. Para mayores detalles sobre el tipo de derechos aplicables en situaciones de emergencia y conflictos armados, consúltese la obra de Melzer (2019: 30). 
organizado y el narcotráfico (véase Kalyvas, 2006; Lessing, 2012; Malesevic, 2010). ${ }^{4}$

\section{Genocidio: el crimen de los crímenes}

Cualquiera sea el tipo de conflicto armado, el marco jurídico aplicable en la conducción de hostilidades es el derecho internacional humanitario (DIH) o derecho internacional de los conflictos armados (DICA), y las violaciones graves a dicha rama del derecho internacional público constituyen crímenes de guerra, que al igual que el crimen de agresión, los crímenes de lesa humanidad y el genocidio, son imprescriptibles (Organización de Naciones Unidas, 1968, 1998, artículos 5 al 8).

De esto cuatro delitos de derecho internacional, se considera al genocidio como el de mayor gravedad: "Existe la noción generalizada de que el genocidio es el crimen de crímenes" (Europa Press, 2020). Esto se explica por el propio significado y alcances del genocidio, entendido como aniquilar, extirpar, liquidar, limpiar; más concretamente, como el exterminio sistemático de un grupo nacional, étnico, racial o religioso (Organización de Naciones Unidas, 1948). Por lo tanto, se erige en cualquiera de una serie de actos cuya comisión tenga por objeto la destrucción total o parcial de ciertos grupos de personas: es esta intención lo que distingue al genocidio de otros crímenes de lesa humanidad o contra la humanidad (Cassese, 2003: 96). De ahí que, para Jacques Billiet, director del Servicio de Información de los Crímenes de Guerra de Francia, el genocidio debe ser concebido como "crimen contra la persona humana" (Autores varios, 1947: 15).

4. El trabajo de Montoya Ramos (2014) presenta evidencias razonables para considerar la situación de violencia en determinadas regiones de México como un conflicto armado no internacional (CANI), lo cual implicaría la activación de un conjunto de protocolos internacionales que regulen el conflicto y minimicen los daños incidentales de la confrontación. 
En su origen gramatical, la palabra proviene de las raíces genos, vocablo griego que significa familia, tribu o raza, y cidio del latín cidere, modificación de caedere: matar; por lo que se trata de una combinación etimológica grecolatina cuya traducción sería: "acción de matar a una familia, tribu o raza".

Se atribuye la paternidad de dicho vocablo al jurista judeo-polaco Raphael Lemkin, quien en 1941 huyó de Polonia ante la invasión de la Wehrmacht; y, después de una breve estadía en los países nórdicos, llegó a refugiarse a Estados Unidos.

En su libro, Lemkin (1944) define el genocidio como el plan coordinado de diferentes acciones cuyo propósito es la destrucción de los cimientos esenciales de la vida de grupos nacionales, con el objetivo de aniquilarlos. Posteriormente esta misma definición sirvió de base a la Convención para la Prevención y Sanción del Delito de Genocidio (Organización de Naciones Unidas, 1948), que el propio Lemkin alienta. ${ }^{5}$

Dicho en otros términos, el genocidio es la negación al derecho de existencia de los grupos humanos, del mismo modo que el homicidio es la negación del derecho a la vida de los seres humanos individuales; de tal negación del derecho o de existencia, se siguen grandes pérdidas para la humanidad, por la privación de las contribuciones culturales y de otro orden representadas por esos grupos humanos, y es contraria a la ley moral y al espíritu y fines de la Organización de las Naciones Unidas (1947: 254-256).

5. Se trata del primer instrumento jurídico internacional en materia de derechos humanos que en su artículo Il consagra:"En la presente Convención, se entiende por genocidio cualquiera de los actos mencionados a continuación, perpetrados con la intención de destruir, total o parcialmente, a un grupo nacional, étnico, racial o religioso, como tal: matanza de miembros del grupo; lesión grave a la integridad física o mental de los miembros del grupo; sometimiento intencional del grupo a condiciones de existencia que hayan de acarrear su destrucción física, total o parcial;medidas destinadas a impedir los nacimientos en el seno del grupo; traslado por fuerza de niños del grupo a otro grupo". 
En resumen, el genocidio constituye un ataque a la diversidad humana como tal, es decir, a una de las características de la "condición humana" sin la cual los términos "humanidad" y "género humano" carecerían de sentido (Arendt, 1967: 387).

Cabe señalar que a la luz del genocidio nazi, Löwy (2003: 42) acuñó el término "barbarie civilizada" de la siguiente forma:

Se podría definir como propiamente moderna a la barbarie que presenta las características siguientes:a) utilización de modernas técnicas (industrialización de la muerte, exterminio en masa gracias a las tecnologías científicas de punta); b) despersonalización de la masacre (poblaciones enteras - hombres y mujeres, niños y ancianos — son "eliminados", con el mínimo contacto personal posible entre los ejecutantes y las víctimas;

c) gestión burocrática, administrativa, eficaz, planificada, "racional" (en términos instrumentales) de actos bárbaros; d) ideologías legitimadoras de tipo moderno:"biológicas","saneadoras", "científicas" (y no religiosas o tradicionalistas).

\section{Conflicto armado}

La guerra, que es "la política con derramamiento de sangre", siguiendo a Karl von Clausewitz (2000: 24), constituye un "verdadero camaleón, por el hecho de que en cada caso concreto cambia de carácter", que presenta retos de la mano de la evolución de la ciencia y la tecnología, cuyo impacto es puesto de relieve en el libro Antidühring por Frederick Engels (Moloeznik, 2018: 73-88, 163-168).

Las guerras del siglo Xxi se caracterizan por su prolongación temporal y por ser mayoritariamente de carácter no internacional (CANI) y, por tanto, afectan sobre todo a la población civil o no combatientes, por ser asimétricas, y por la diversidad y complejidad de grupos armados no estatales, entre otras notas esenciales (véase Münkler, 2003). 
En lo que respecta a las condiciones de un conflicto armado, en particular de carácter no internacional (CANI), el Documento de opinión del CICR 6 - publicado en 2008define a los CANI como

[...] enfrentamientos armados prolongados entre fuerzas armadas gubernamentales y las fuerzas de uno o más grupos armados, o entre estos grupos, que surgen en el territorio de un Estado [parte en los Convenios de Ginebra]. El enfrentamiento armado debe alcanzar un nivel mínimo de intensidad y las partes que participan en el conflicto deben poseer un mínimo de organización (Comité Internacional de la Cruz Roja, 2008: 10).

Esto es, las condiciones para la existencia de un CANI son las siguientes:

Umbral de intensidad: es necesario que el enfrentamiento alcance una situación de "violencia armada prolongada" entre un Estado y grupos armados organizados, o bien entre esos grupos, que no pueda controlarse mediante la actividad policial habitual en tiempo de paz, es decir que imponga la intervención de las fuerzas armadas, a la sazón instrumento coercitivo de máxima intensidad de uso de la fuerza del Estado como detentador del monopolio legítimo de la fuerza.

Entre los elementos indicativos para evaluar el nivel de dicha intensidad destacan: el número de enfrentamientos y la duración e intensidad de cada uno de los mismos; el tipo de medios (armas y equipamiento militar utilizado); el número y el calibre de las municiones utilizadas; el número de combatientes y los tipos de fuerzas que participan en los enfrentamientos; el número de bajas; así como la extensión de la destrucción material y el número de civiles expulsados de las zonas de combate.

Umbral de organización: sin un nivel mínimo de organización es imposible desarrollar operaciones militares coor- 
dinadas y sostenidas, así como garantizar el cumplimiento general del DIH.

Entre los factores indicativos se incluyen elementos tales como: la existencia de una cadena de mando y de normas y mecanismos disciplinarios dentro del grupo; contar con un cuartel general; ejercer cierto control territorial; tener capacidad para aprovisionarse de armamento y de otro equipamiento; estar en condiciones de reclutar personal y de desarrollar entrenamiento militar; poder planificar, coordinar y ejecutar operaciones militares, incluyendo movimientos de tropas y logística; definir una estrategia militar unificada y emplear tácticas militares; contar con un vocero oficial; y, tener la capacidad para negociar y celebrar acuerdos de paz o de cese al fuego, entre otros.

Actualmente a los conflictos armados se los conoce como de cuarta generación, también denominados guerras híbridas, término acuñado por James N. Mattis y Frank G. Hoffman (2005), oficiales del Cuerpo de Infantería de Marina de Estados Unidos (USMC).

\section{Otras situaciones de violencia}

El DIH reconoce también la existencia de niveles de violencia más bajos, tal como se desprende del párrafo 2 del artículo 1 del Protocolo II adicional a los Convenios de Ginebra de 1949, que a la letra reza: "El presente Protocolo no se aplicará a las situaciones de tensiones internas y de disturbios interiores, tales como los motines, los actos esporádicos y aislados de violencia y otros actos análogos, que no son conflictos armados" (Comité Internacional de la Cruz Roja, 1977).

Asimismo, en dichas situaciones que no alcanzan el umbral de conflicto armado y que se agrupan en lo que el Comité Internacional de la Cruz Roja llama "otras situaciones de violencia" (osv): 
[...] todo empleo de la fuerza u otra autoridad que ejerzan los Estados en contra de grupos o individuos que se encuentren dentro de su jurisdicción son regidos por el derecho de los derechos humanos, mientras que los hechos de violencia o daños que ocasionen dichos grupos o individuos quedará en el ámbito del mantenimiento de la ley y el orden y, por lo tanto, estarán regulados por el derecho nacional (Comité Internacional de la Cruz Roja, 20I5b: 22 y 23).

De ahí la importancia de dar cuenta de las características esenciales de las osv, que se entienden a partir del fenómeno de los disturbios y tensiones internas.

Los disturbios se definen como situaciones de enfrentamiento que presentan cierto carácter de gravedad o duración, con una profunda perturbación del orden interior y que comprenden actos de carácter violento, tales como reuniones, concentraciones, manifestaciones reivindicativas, huelgas de ámbito nacional, motines y golpes de Estado, en cuyo contexto las autoridades gubernamentales se enfrentan a grupos de personas o manifestantes (Costas Trascasas, 2005: 58). Se trata, por ejemplo, de motines mediante los cuales grupos de personas manifiestan abiertamente su oposición, su descontento o sus reivindicaciones, o también de actos aislados y esporádicos de violencia. Puede tratarse, incluso, de luchas de facciones entre ellas o contra el poder establecido (Comité Internacional de la Cruz Roja, 1988: 9 y ss.). La respuesta estatal suele ser el empeñamiento de gran número de fuerzas policiales, de seguridad o militares, para restablecer la ley y el orden interno deteriorados, y suele traer aparejado un elevado número de víctimas, como resultado de las medidas represivas.

Las situaciones de tensión se caracterizan porque no se producen o no llegan a producirse enfrentamientos violentos propiamente dichos; por lo que las autoridades suelen recurrir al uso de la fuerza y al establecimiento de legislación de excepción con carácter preventivo, para reducir 
la tensión política, social, económica o étnica (de Rover, 1988: 214, 440 y 444). Su origen descansa, principalmente, en problemas económicos que pueden provocar huelgas generales, con riesgo de incumplimiento de los servicios mínimos; así como en problemas sociales que produzcan protestas generalizadas que puedan desembocar en violencia; en problemas derivados de situaciones catastróficas que pueden degenerar en pillaje y saqueos; en problemas sanitarios que desencadenen epidemias, alarmas; y hasta en el monopolio de medicamentos o recursos vitales, en detrimento de la comunidad.

En síntesis, los disturbios interiores se presentan cuando el Estado utiliza la fuerza armada para mantener el orden, sin que haya conflicto armado; en tanto que la existencia de tensiones internas responde al empleo de la fuerza como una medida preventiva para mantener el respeto de la ley y el orden.

Los osv no son conflictos armados y, por ende, no alcanzan el umbral de aplicación del DIH, sino del derecho internacional de los derechos humanos (DIDH) y el derecho interno de cada Estado soberano, aunque el Comité Internacional de la Cruz Roja reconoce que los osv suelen presentar efectos humanitarios muchas veces de mayor magnitud y devastación que los conflictos armados propiamente dichos.

Por último, cabe destacar que en actividades de aplicación de la ley, el DIDH establece que la fuerza letal sea empleada solo como último recurso, cuando otros medios no resultan eficaces o no garantizan que se alcance el objetivo de una operación de aplicación de la ley (Comité Internacional de la Cruz Roja, 2015a: 19). ${ }^{6}$ 


\section{Terrorismo}

Si bien la comunidad internacional condena al terrorismo en todas sus formas y manifestaciones, al reconocerlo como una de las amenazas más graves a la paz y la seguridad internacionales, no existe una definición universal, salvo su caracterización como actividad delictiva grave motivada por un objetivo en particular (Organización de Naciones Unidas, 2006).

En contraste, sí existe un consenso sobre su naturaleza, al ser concebido como un fenómeno sociopolítico que apela a la violencia y a la amenaza de la misma, con la intención de alterar los comportamientos sociales e institucionales, generando reacciones como la ansiedad, la incertidumbre, el miedo o la intimidación, mediante la realización de acciones violentas que persiguen provocar efectos psíquicos desproporcionados respecto a las consecuencias materiales causadas. El recurso a dicha violencia persigue la consecución de objetivos que generalmente son de tipo político y religioso (Autores varios, 2013).

También es definido como un método de violencia "indiscriminada y sin control" (Gasser, 2002), con la finalidad de causar terror aplicado fundamentalmente a los no combatientes. De conformidad con Swinarski (2003: 534), uno de los primeros intentos por definirlo es el Convenio para la Prevención y Castigo del Terrorismo de 1937, en cuya virtud terrorismo eran aquellos "actos criminales dirigidos contra un Estado con la intención o procurando crear un estado de terror particularmente a las personas o grupo de personas o al público en general". Esto significa que el terrorismo es un flagelo que se caracteriza por actos indiscriminados de violencia que suelen perpetrarse sobre la población civil, como un medio para lograr determinados objetivos.

Como quiera que sea, el desafío descansa en cómo conciliar su tratamiento efectivo para salvar vidas humanas con 
el respeto de los derechos humanos, es decir, cómo evitar el sacrificio de los derechos fundamentales en el altar de la seguridad. El marco jurídico internacional y los instrumentos que se desprenden de los mismos constituyen el primer escalón de protección contra la barbarie; el Estado-nación como detentador del monopolio legítimo de la fuerza no puede, bajo circunstancia alguna, utilizar los mismos métodos que los terroristas, porque de lo contrario pondría en entredicho la ética y principios sobre los que se sustenta.

\section{Violencia criminal organizada}

Mientras que la violencia criminal organizada es un tipo de violencia particular (véase Schedler, 2015). En primer lugar, los actores de la violencia son grupos o colectivos altamente estructurados, con autonomía en su operatividad y un cierto grado de cohesión identitaria (Paoli, 2003). Particularmente estos grupos o colectivos desarrollan actividades ilegales o proscritas, de ahí que sean criminales o delincuentes. En segundo lugar, los motivos de la violencia criminal organizada son principalmente económicos, aunque suelen combinarse con cuestiones ideológicas, religiosas o políticas, según el grupo de que se trate (Maltz, 2010). Tratándose del caso mexicano, no es evidente que estos colectivos tengan motivaciones políticas, al menos no se ha comprobado que quieran cambiar el sistema político $u$ ocupar posiciones políticas, pero parece que sí tienen intenciones de incidir en algunos sectores de la administración pública. En tercer lugar, el uso de la violencia por parte de los grupos del crimen organizado responde a mecanismos y procesos generados para la administración de la violencia. Esto quiere decir que la violencia es selectiva, orientada a resultados, de carácter estratégico, y que existen mecanismos moderadamente institucionalizados para la conducción de la violencia (Koerin, 1978; Schelling, 2010). Por último, 
la violencia criminal organizada comparte características de los conflictos armados no internacionales (Albanese, 2007; Kaldor, 2001; Kalyvas, 2015), por lo que la lógica de su ejercicio debe buscarse también en este contexto.

La violencia criminal organizada es una forma de violencia colectiva. El uso de la violencia en este tipo es instrumental, lo que quiere decir que personas que se identifican a sí mismas como miembros de un grupo utilizan la violencia contra otro grupo o conjunto de individuos para lograr objetivos políticos, económicos o sociales (oPs, 2003: 235). Por tanto, el supuesto es que los cárteles de la droga, la delincuencia organizada ${ }^{7}$ o los actores de la violencia criminal utilizan racionalmente la violencia en función de objetivos particulares. Lo cual es cierto y así ha sido demostrado por diversos estudios sobre la delincuencia organizada (véase Albanese, 2007; Beittel, 2015; Kaldor, 2001; Kalyvas, 2006; Paoli, 2014).

Sin embargo, los actores de la violencia colectiva no siempre la ejercen racionalmente o como medio para un fin. Como han puesto al desnudo algunos estudiosos, el ejercicio de la violencia por parte de algunos colectivos se ha constituido, en algunos momentos y contextos específicos, en un fin en sí mismo (Bernstein, 2015; Blair, 2009; Kaldor, 2001; Reguillo, 2011).

\section{Conclusiones: sobre los paradigmas de la violencia}

La violencia constituye un fenómeno por demás complejo a la vez que es una constante histórica. Esto explica que

7. De acuerdo con la Convención de las Naciones Unidas contra la Delincuencia Organizada Transnacional, la delincuencia organizada se entiende como "un grupo estructurado de tres o más personas [...] que actúe concertadamente con el propósito de cometer uno o más delitos graves o delitos tipificados [...] con miras a obtener, directa o indirectamente, un beneficio económico u otro beneficio de orden material" (Oficina de las Naciones Unidas contra la Droga y el Delito, 2004: 5). 
diversos pensadores y tratadistas hayan intentado definirla, interpretarla y clasificarla. Actualmente la violencia da al traste con el más sagrado de los derechos humanos, el derecho a la vida. En particular, es la violencia colectiva - aquella que afecta a grandes colectivos sociales- la responsable del mayor número y tasa de defunciones del orbe. No obstante su importancia y peso, no existe una definición de la violencia aceptada universalmente, así como tampoco una teoría que sea capaz de explicar todas las formas de violencia.

Tal como se pone de relieve, es posible distinguir dos concepciones generales de la violencia: una restringida y otra ampliada. La concepción restringida de la violencia hace hincapié en el daño físico, a la sazón su característica objetiva y medible. De manera tal que la muerte ajena a causas naturales se constituye como el indicador global y predilecto de la violencia, y se pone especial énfasis en las consecuencias y la gravedad del daño sobre las víctimas. La concepción ampliada de la violencia reconoce un conjunto más amplio de actos que implican ejercicios intencionales de poder contra individuos o colectivos, que no se expresan solo en daños físicos, pero que reproducen relaciones sistemáticas de desigualdad, exclusión, subordinación u otras formas sutiles de victimización y coerción.

En cuanto a la clasificación de la violencia, la concepción restringida propone tomar en consideración cuatro criterios: a) los actores de la violencia; b) los motivos de la violencia; c) el grado de organización de la violencia, y d) los escenarios de la violencia.

La evidencia y diversos estudios soportados en datos duros demuestran que el genocidio y otros crímenes de lesa humanidad, los crímenes de guerra, los actos esporádicos y aislados de violencia —otras situaciones de violencia-, el terrorismo y la violencia criminal organizada son las modalidades de la violencia responsables de la mayoría de 
las muertes violentas en el orbe que ponen en entredicho el pacto social y, por ende, la seguridad comunitaria.

Así, para la Organización Mundial de la Salud, las formas de violencia colectiva más reconocidas son los conflictos armados, el terrorismo, las situaciones complejas de emergencia, el genocidio, la represión, las desapariciones, la tortura y las masacres (oPs, 2003: 235), a las que se suma el accionar de la violencia criminal, traducido en ejecuciones y enfrentamientos armados.

En este marco, distintos grupos hacen uso de la violencia colectiva, como el Estado, los grupos guerrilleros, los terroristas, la delincuencia organizada y algunos movimientos sociales. En especial, los grupos delictivos organizados se caracterizan por un uso extensivo de distintas formas de violencia colectiva (Albanese, 2000, 2007; Shaw, 2009).

La respuesta estatal sin trastocar los principios fundamentales del Estado de derecho — corazón del ordenamiento penal, procesal y constitucional de los sistemas democráticos - se erige en un reto insoslayable (López Garrido, 1987: I y II), que se traduce en el delicado equilibrio entre la vigencia plena de los derechos humanos (en especial, el derecho a la vida) y la violencia institucionalizada.

Como quiera que sea, el lector se encuentra frente a la violencia como principal desafío social en tanto que, como subraya el padre jesuita Ignacio Martín Baró, "es un fenómeno donde lo social se vuelve individual y donde lo personal se traduce en construcción social" (Garavito, 2005: 43), cuya atención se confía al Estado-nación en tanto árbitro y orientador de los conflictos sociales y como detentador del monopolio legítimo de la fuerza. 
Bibliografía
Albanese, J. S. (2000). The Causes of Organized Crime: Do Criminals Organize Around Opportunities for Crime or Do Criminal Opportunities Create New Offenders? Journal of Contemporary Criminal Justice, I6(4): 409-423. https://doi.org/I 0.1 I 77// 043986200016004004

_. (2007). Organized Crime in OurTimes. (5 edición). Lexis Nexis/Anderson Publishing.

Arendt, H. (1967). Eichmann en Jerusalén: Un estudio sobre la banalidad del mal. Barcelona: Lumen.

-. (2006). Sobre la violencia. Alianza Editorial.

Arteaga Botello, N. (2007). Repensar la violencia. Tres propuestas para el siglo XXI. Trayectorias, IX(23): 43-54.

Autores varios. (1947). Campos de concentración. El crimen más grande contra el género humano. Editorial Problemas.

Autores varios. (2013). Diccionario de inteligencia y seguridad. Gobierno de España-Ministerio de la Presidencia/LID Editorial Empresarial.

Bagley, B. M., y Rosen, J. D. (Eds.). (2015). Drug Trafficking, Organized Crime, and Violence in the Americas Today. University Press of Florida.

Beittel, J. S. (2015). Mexico: Organized Crime and Drug Trafficking Organizations (núm. R4I576). Congressional Research Service (CRS) Reports. https://www.fas.org/ sgp/crs/row/R4I576.pdf

Benjamin,W. (1995). Para una crítica de la violencia. Leviatán. Bernstein, R.J. (20I5). Violencia. Pensar sin barandillas. Gedisa Editorial.

Blair, E. (2009). Aproximación teórica al concepto de violencia:Avatares de una definición. Política y Cultura, núm. 32, pp. 9-33.

Bourdieu, P., y Passeron, J.-C. (Eds.). (200I). Fundamentos de una teoría de la violencia simbólica. La reproducción. Elementos para una teoría del sistema de enseñanza (pp. 15-85). Editorial Popular. 
Cassese,A. (2003). International Criminal Law. Oxford: Oxford Bibliografía University Press.

Collins, R. (2008). Violence. A micro-sociological theory. Princeton University Press.

- (2009). Micro and Macro Causes of Violence. International Journal of Conflict and Violence, 3(I): 9-22.

Comité Internacional de la Cruz Roja. (1977). Protocolo II adicional a los Convenios de Ginebra de 1949 relativo a la protección de las víctimas de los conflictos armados sin carácter internacional. Comité Internacional de la Cruz Roja. https://www.icrc.org/es/doc/resources/documents/misc/protocolo-ii.htm

- (1988). Actividades de protección y de asistencia del CICR en las situaciones que no abarca el derecho internacional humanitario. Revista Internacional de la Cruz Roja, núm. 85.

- (1998). Comentario del Protocolo adicional II a los Convenios de Ginebra de 1949. Comité Internacional de la Cruz Roja. https://www.icrc.org/es/doc/resources/ documents/misc/comentario-protocolo-ii.htm

—_. (2008). ¿Cuál es la definición de "conflicto armado"según el derecho internacional humanitario? Comité Internacional de la Cruz Roja. http://www.icrc.org/spa/resources/ documents/article/other/armedconflict-article- 170308. htm

- (20I5a). Cuarto informe: xxxII Conferencia Internacional de la Cruz Roja y de la Media Luna Roja. Ginebra:Comité Internacional de la Cruz Roja. https://www.icrc.org/ es/document/el-derecho-internacional-humanitario-y-los-desafios-de-los-conflictos-armados

- (20I5b). Violencia y uso de la fuerza. Ginebra: Comité Internacional de la Cruz Roja. https://www.icrc.org/ es/doc/assets/files/other/violencia-y-uso-de-la-fuerza_ (web).pdf 
Costas Trascasas, M. (2005). El marco legal y reglamentario aplicable al restablecimiento del orden público y la protección de las personas en situaciones de disturbios y tensiones internas. En: Comité Internacional de la Cruz Roja, e Instituto Interamericano de Derechos Humanos, Informe sobre el uso de la fuerza y la protección de las personas en situaciones de disturbios y tensiones internas en América Latina y el Caribe. Informe de una reunión de expertos.

Crettiez, X. (2009). Las formas de la violencia. Waldhuter Editores.

De Rover, C. (1998). Servir y proteger. Derecho de los derechos humanos y derecho humanitario para las fuerzas de policía y de seguridad. Comité Internacional de la Cruz Roja. https://www.icrc.org/spa/resources/documents/ publication/p0698.htm

Europa Press. (2020). Genocidio, el "crimen de crímenes" al que ningún país y ninguna región es "inmune”. Europa Press. https://www.europapress.es/internacional/noticia-genocidio-crimen-crimenes-ningun-pais-ninguna-region-inmune-20200407 I03 I0 I.html

Fanon, F. (1973). Piel negra, máscaras blancas. Abraxas.

Forst, B. (2008). Terrorism, crime and public policy. Cambridge University Press.

Galtung,J. (1998). Tras la violencia, 3R: Reconstrucción, reconciliación, resolución. Afrontando los efectos visibles e invisibles de la guerra y la violencia. Bakeaz/Gernika Gogoratuz.

- (2009). Cultural Violence. Journal of Peace Research, 27(3): 29|-305.

Garavito Fernández, M.A., y otros (2005). Las violencias en Guatemala, algunas perspectivas. FLACSO/UNESCO.

García-Sílberman, S., y Ramos Lira, L. (2000). Medios de comunicación y violencia. Fondo de Cultura Económica.

Gasser, H. (2002). Actos de terror, "terrorismo" y derecho internacional humanitario. Revista Internacional de la Cruz

\section{4}


Roja.https://www.icrc.org/es/doc/resources/documents/ Bibliografía misc/5ted8g.htm

Gros, F. (2006). États de violence. Essai sur la fin de la guerre. Gallimard.

Imbusch, P., Misse, M., y Carrión, F. (20I I).Violence research in Latin America and the Caribbean:A literature review. International Journal of Conflict and Violence, 5(I): 87-I54. Kaldor, M. (200I). Las nuevas guerras.Violencia organizada en la era global.Tusquets Editores.

Kalyvas, S. N. (2006). The Logic of Violence in Civil War. Cambridge University Press.

- (2015). How CivilWars Help Explain Organized Crimeand How They Do Not.Journal of Conflict Resolution, 59(8): I5 I7-I540. https://doi.org//0.1 I77/00220027/5587 I0 I

Koerin, B. (1978). Violent Crime: Prediction and Control. Crime \& Delinquency, 24(I): 49-58. https://doi. org/I0.1 I 77/00 I I I2877802400I05

Krug, E. G., Dahlberg, L. L., Mercy, J.A., Zwi,A. B.,y Lozano, R. (Eds.). (2003). Informe mundial sobre la violencia y la salud, núm. 588. Organización Panamericana de la Salud-Oficina Regional para las Américas.

Lemkin, R. (1944). Axis Rule in Occupied Europe: Laws of Occupation, Analysis of Government, Proposals for Redress. Carnegie Endowment for International Peace.

Lessing, B. (20/2). The logic of violence in criminal war: Cartel-State conflict in Mexico, Colombia, and Brazil. Berkeley: University of California.

- (20I3). Violent corruption and violent lobbying: Logics of cartel-State conflict in Mexico, Brazil and Colombia. Center for International Security and Cooperation. http://cisac. stanford.edu/publications/24162

López Garrido, Diego. (1987). Terrorismo, política y derecho. La legislación antiterrorista en España, Reino Unido, República Federal de Alemania, Italia y Francia. Alianza Editorial. 
Löwy, M. (2003). Las formas modernas de la barbarie. Metapolítica: Las Barbaries de la Civilización, 28(7).

Maldonado Aranda, S. (20I2). Transición política, seguridad y violencia en México: Radiografía de la lucha antidrogas en Michoacán. El prisma de las seguridades en América Latina. Escenarios regionales y locales (pp. II5-I38). Clacso.

Malesevic, S. (2010). The sociology of war and violence. Cambridge University Press.

Maltz, M. D. (2010). On defining "organized crime": The development of a definition and tipology. En F.Varese (Ed.), Organized Crime (pp. 66-75). Routledge.

Marina,J.A. (2004). Crónicas de la ultramodernidad. Quintento. Mattis, J. N., y Hoffman. (2005). Future Warfare:The Rise of HybridWars. U. S. Naval Institute. https://www.usni.org/ magazines/proceedings/2005/november/future-warfarerise-hybrid-wars

Mbembe, A. (20II). Necropolítica. Melusina. https://doi. org/10.1007/s I3398-0|4-0173-7.2

Melzer, N. (20/9). Derecho internacional humanitario. Una introducción integral. Comité Internacional de la Cruz Roja. https://www.casede.org/index.php/biblioteca-casede-2-0/justicia/492-derecho-internacional-humanitario-una-introduccion-integral

Moloeznik, M. P. (20|8). Tratado sobre pensamiento estratégico-militar Enseñanzas para el sistema de defensa de México. Colectivo de Análisis de la Seguridad en Democracia, A. C. (Casede). http://www.casede.org/ index.php/biblioteca-casede-2-0/defensa-y-fuerzas-armadas/fuerzas-armadas-mexicanas/4 I 0-tratado-sobre-pensamiento-estrategico-militar

Montoya Ramos, I. (20I4). Criminalidad organizada y conflicto armado no internacional. En N.T. Bravo Aguilar y J. G. García Murillo (Eds.), Balance e impacto de las políticas públicas federales en materia de seguridad bajo la gestión

\section{6}


de Felipe Calderón Hinojosa (2006-20 I 2). Universidad de Bibliografía Guadalajara.

Münkler, H. (2003). Las guerras del siglo xxI. Revista Internacional de la Cruz Roja. https://www.icrc.org/es/doc/ resources/documents/misc/5tedfy.htm

Oficina de las Naciones Unidas contra la Droga y el Delito. (2004). Convención de las Naciones Unidas contra la Delincuencia Organizada Transnacional y sus protocolos.

Organización de Naciones Unidas (ONU). (1947). Resolución 96 (I) de la Asamblea General de la onu. United Nations Yearbook, 1946-47.

- (1948). Convención para la Prevención y la Sanción del Delito de Genocidio. Comité Internacional de la Cruz Roja (CICR). https://www.icrc.org/es/doc/resources/documents/misc/treaty-1948-conv-genocide-5tdm6h.htm

- (1968). Convención sobre la imprescriptibilidad de los crímenes de guerra y de los crímenes de lesa humanidad. Organización de Naciones Unidas. https://www.ohchr. org/sp/professionalinterest/pages/warcrimes.aspx

- (1998). Estatuto de Roma de la Corte Penal Internacional. Organización de Naciones Unidas. http://www.oas. org/36ag/espanol/doc_referencia/Estatuto_Roma.pdf

- (2006). Resolución A/RES/60/288 de la Asamblea General, Estrategia global de las Naciones Unidas contra el terrorismo. Organización de Naciones Unidas. https://undocs.org/ es/a/res/60/288

Paoli, L. (2003). Mafia Brotherhoods. Organized Crime, Italian Style. Oxford University Press.

-. (Ed.). (20I4). The Oxford Handbook of Organized Crime. Oxford University Press.

Reguillo, R. (20II). La narcomáquina y el trabajo de la violencia:Apuntes para su decodificación. Emisférica. http:// hemisphericinstitute.org/hemi/es/e-misferica-82/reguillo _..(2014). La narcomáquina ya no necesita Chapos. Revista Anfibia, Pp. I-5. 
Restrepo, J.A., y Aponte, D. (Eds.). (2009). Guerra y violencias en Colombia. Herramientas e interpretaciones. Editorial Pontificia Universidad Javeriana.

Rouquié, A. (1989). América Latina. Introducción al extremo Occidente. Siglo XXI Editores.

Schedler, A. (20I5). En la niebla de la guerra: Los ciudadanos ante la violencia criminal organizada. Centro de Investigación y Docencia Económicas (CIDE).

Schelling, T. C. (20I0). What is the bussiness of organized crime? En F.Varese (Ed.), Organized Crime (pp. 26I-273). Routledge.

Schinkel,W. (20 I0). Aspects of Violence: A Critical Theory. Palgrave Macmillan.

Schmitt, C. (20I4). El concepto de lo político:Texto de 1932 con un prólogo y tres corolarios. Alianza Editorial.

Shaw, M. (2009). Conceptual and Theoretical Frameworks for Organised Violence. International Journal of Conflict and Violence, 3(I): 97-I06.

Siegel, D., y Bunt, H.van de (Eds.). (20I2). Traditional Organized Crime in the Modern World. Responses to Socioeconomic Change. Springer.

Sofsky, W. (2004). Tiempos de horror. Amok, violencia, guerra. Siglo XXI Editores.

Sorel, G. (2004). Reflections on Violence. Cambridge University Press.

Swinarski, C. (2003). Terrorismo en el derecho internacional público (pp. 533-547). EnValladares, G.P. (Comp.), Derecho internacional humanitario y temas de áreas vinculadas. Lexis Nexis Abeledo Perrot.

Tilly, C. (2006). Guerra y construcción del Estado como crimen organizado. Revista Académica de Relaciones Internacionales, núm. 5, pp. I-26.

- (2008). The Politics of Collective Violence. Cambridge University Press.

Valencia, S. (2010). Capitalismo Gore. Melusina. 
Von Clausewitz, K. (20 I0). De la guerra. Biblioteca Virtual UniBibliografía versal. https://www.biblioteca.org.ar/libros/ I5374I.pdf

Wieviorka, M. (2009). Violence. A New Approach. Sage.

Winkler, C. K. (2006). In the Name of Terrorism. Presidents on Political Violence in the Post-World War II Era. State University of New York Press.

World Health Organization (WHO).(20I4). Global Status Report on Violence Prevention 20/4. https://doi.org/10.1007/ sl3398-0|4-0173-7.2

Zimbardo, P. (2008). El efecto Lucifer: El porqué de la maldad. Paidós.

Zizek, S. (1999). El acoso de las fantasías. Siglo XXI Editores. . (2009). Sobre la violencia. Seis reflexiones marginales. Paidós. 
